\title{
The case of the missing aneurysm
}

\section{Ian C Duncan FFRad(D)SA}

\section{Pieter A Fourie MMedRad (D)}

Unitas Interventional Unit PO Box 14031

Lytitelton 0410

\section{Introduction}

Spontaneous thrombosis of an intracranial aneurysm following acute rupture is well described. This can result in a false-negative cerebral angiogram if done early after rupture. We describe a case showing disappearance and spontaneous reappearance of an aneurysm on serial angiography in a patient with multiple intracranial aneurysms following subarachnoid haemorrhage. Another important feature of this case was the lack of correlation between the findings on sectional imaging and the initial angiogram, later solved by the reappearance of the occult aneurysm.

\section{Case report}

A 36-year-old male patient presented to a peripheral hospital with an acute subarachnoid haemorrhage (SAH). An unenhanced computed tomographic (CT) scan done shortly after admission demonstrated a focal area of haemorrhage related to the medial aspect of the right sylvian fissue with intraparenchymal breakthrough haemorrhage into the region of the anterior perforating substance and further breakthrough into the medial aspect of the right temporal lobe (Fig. 1a). He was later transferred to the Pretoria Academic Hospital where a digital subtraction cerebral arteriogram was performed. This demonstrated mild arterial spasm in both distal internal carotid arteries, more on the right, and small aneurysms at the left carotid bifurcation and at the origin of the left superior cerebellar artery from the basilar artery (Figs 1b - d). No other aneurysms were seen at this stage. The opinion given at that stage was that the haemorrhage may have arisen from the posterior circulation aneurysm and the patient was therefore referred to the Unitas Interventional Unit for possible endovascular treatment.

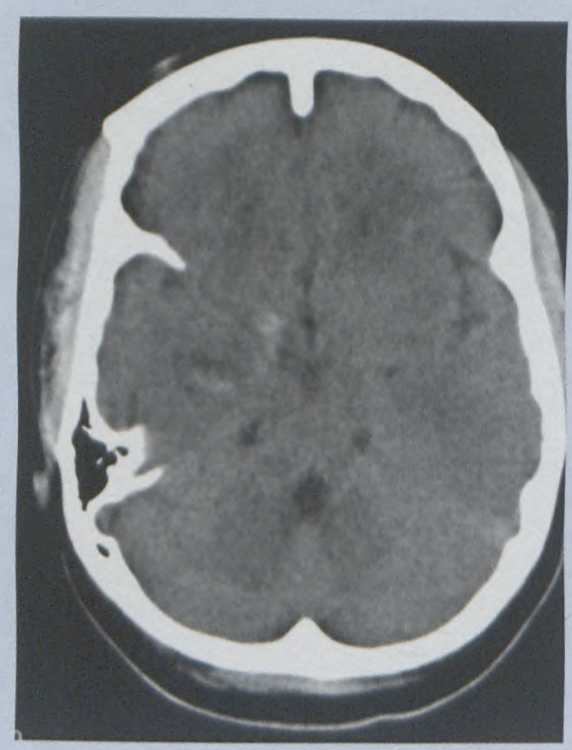

Fig. 1a. Unenhanced axial CT scan showing haemorrhage in the region of the anterior perforating substance and in the medial aspect of the right temporal lobe.

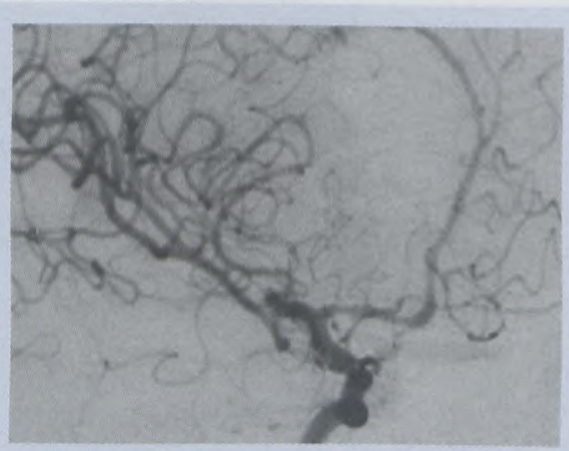

Fig. 1b. Selective right internal carotid digital subtraction arteriogram showing the carotid termination and proximal anterior cerebral artery. No aneurysm is seen here.

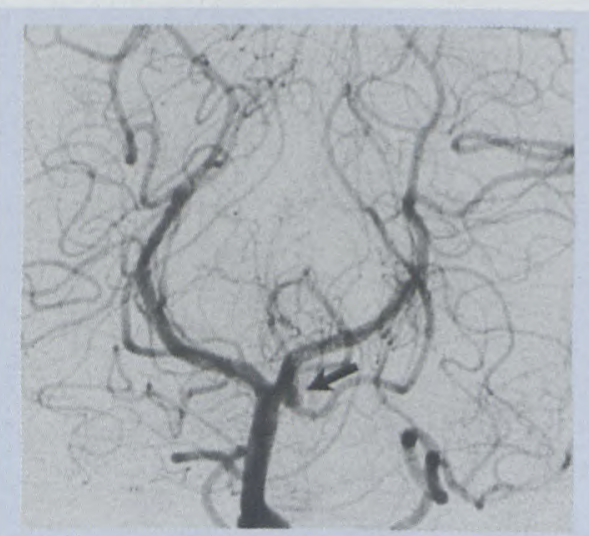

Fig. 1c. Selective right vertebral arteriogram shows the small left-sided superior cerebellar aneurysm (arrow).

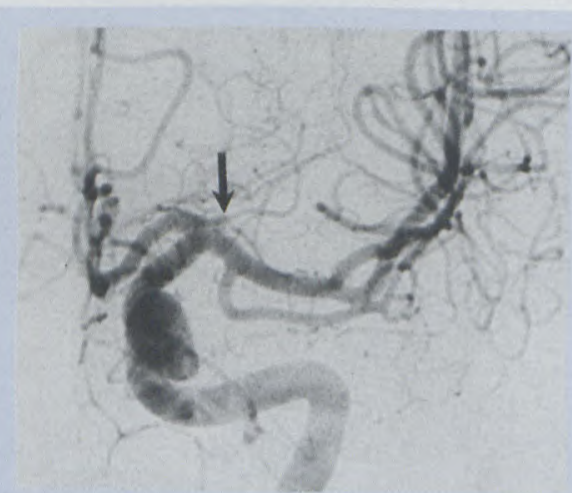

Fig. 1d. Selective left intemal carotid arteriogram showing the small left carotid bifurcation aneurysm (arrow).

We performed the second angiogram 6 weeks after the initial haemorrhage. The patient had recovered well from the initial SAH and was clinically stable. Our impression was that the position of the haemorrhage 
on the original CT scan did not correlate with the position of the aneurysms shown on the original angiogram. The repeat angiogram now showed the presence of a 5 $6 \mathrm{~mm}$ diameter aneurysm arising from the proximal part of the Al segment of the right anterior cerebral artery, a mirror-type aneurysm to the smaller left-sided bifurcation aneurysm (Figs 2a, b). 3D rotational angiograms of the basilar and right internal carotid arteries were performed (Figs 2c, d). The smaller basilar aneurysm was broad-based, measuring $2 \mathrm{~mm}$ in diameter, and incorporating the origin of the left superior cerebellar artery itself (Fig. 2e). The right 'bifurcation' aneurysm was shown to arise from the postero-superior wall of the proximal aspect of the Al segment of the right anterior cerebral artery rather than the bifurcation itself.

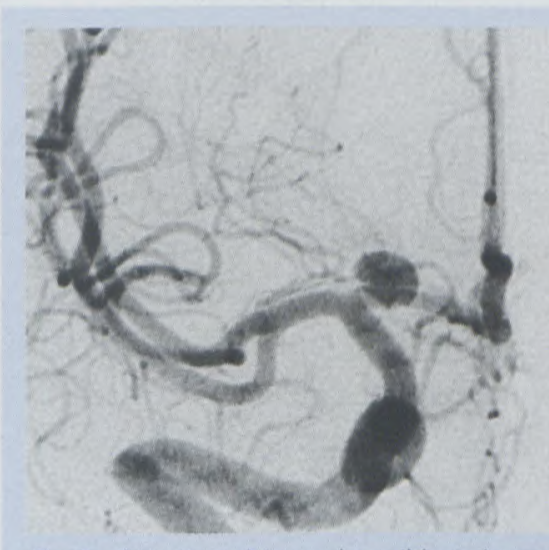

Fig. 2a. Selective left internal carotid arteriogram, Towne's projection done 6 weeks after the SAH, now showing the aneurysm on the $A 1$ segment.

Retrospective review of the original angiogram showed a shallow convex negative filling defect along the upper aspect of the proximal $\mathrm{Al}$ segment at the site of the neck of the aneurysm, probably produced by adherent thrombus at the neck of the aneurysm (Fig. 3). The presence of an

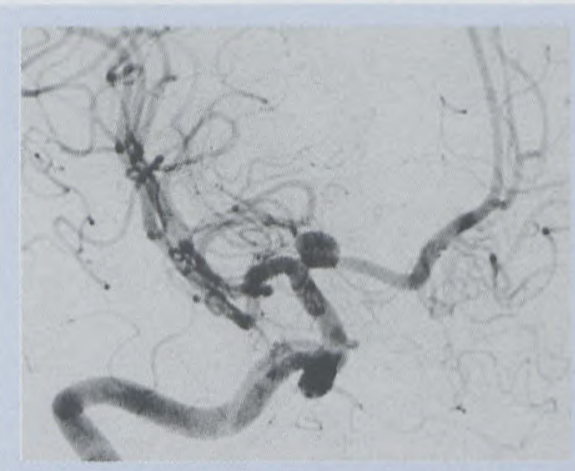

Fig. 2b. Same vessel, right anterior oblique view.

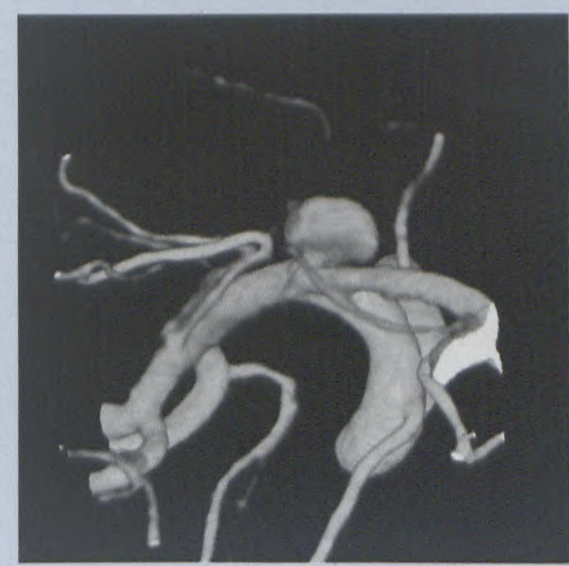

Fig. 2c. 3D Rotational angiogram, anterosuperior projection, showing the neck of the aneurysm arising from the posterosuperior aspect of the right A1 segment.

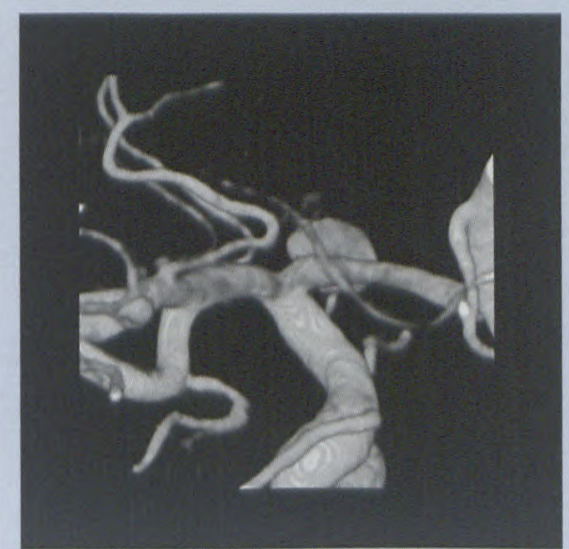

Fig. 2d. 3DRA, anteroinferior projection, again showing the carotid bifurcation region.

aneurysm in this position also explained the greater degree of vasospasm seen in the right supraclinoid ICA on the original arteriogram. Despite the newly-detected aneurysm

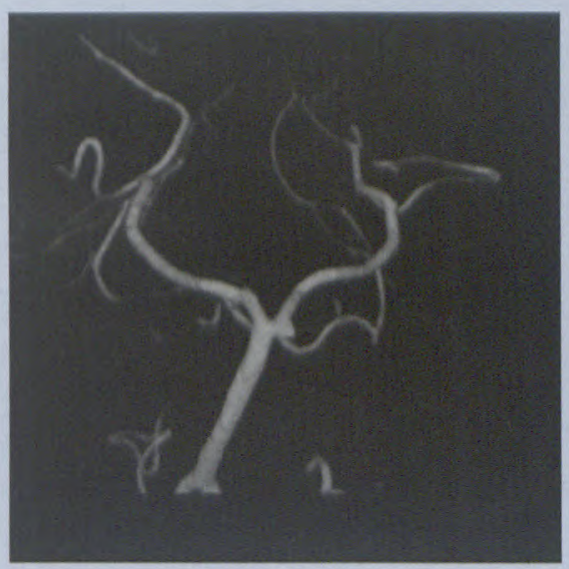

Fig. 2e. 3DRA, Towne's projection, showing detail of the superior cerebellar aneurysm.

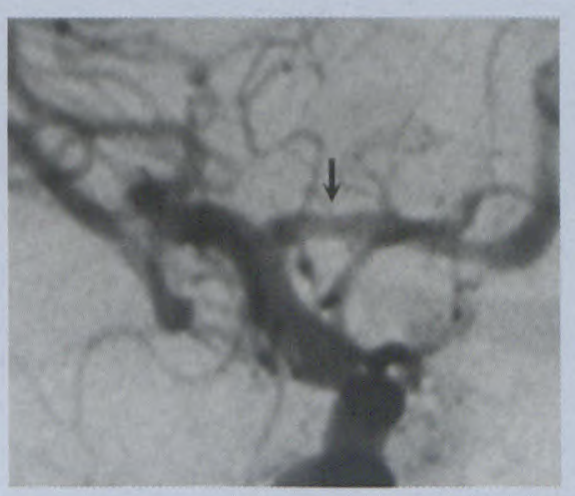

Fig. 3. Retrospective analysis of the anatomy of the right $A 1$ segment on the initial angiogram shows a shallow convex filling defect at the opening of the aneurysm due to thrombus (arrow).

being highly suitable for endovascular treatment, it was decided to refer the patient for later surgical clipping.

\section{Discussion}

Between $10 \%$ and $20 \%$ of cerebral angiograms performed on patients with acute subarachnoid haemorrhage (SAH) will show no evidence of any aneurysm or other causative lesion (Table I). ${ }^{1,2}$ Non-aneurysmal causes of SAH are listed in Table II. Spontaneous thrombosis of a ruptured intracranial aneurysm has been described in $9 \%-13 \%$ of cases in autopsy series. ${ }^{3}$ Recannalisation of previously thrombosed aneurysms 
Table I. Technical reasons for failure to demonstrate an aneurysm at angiography

1. Aneurysm-related:

Spontaneous thrombosis

Vasospasm

Parent vessel thrombosis

2. Angiographic factors

Inadequate number of views (projections per vessel)

Inadequate contrast opacification

Inadequate number of vessels studied

Interpretive error (operator dependence)

Motion artefact (restless patient)

Inadequate or outdated angiographic equipment

Vascular washout effects

Failure to demonstrate the communicating arteries. has also previously been described. ${ }^{4}$ Spontaneous thrombosis may be related to flow stasis, hypercoagulability, spasm, endothelial injury or antifibrinolytic activity. For cases with an initial negative cerebral angiogram in the setting of acute $\mathrm{SAH}$, repeat angiography has been advocated between 3 and 6 weeks after the initial haemorrhage. This has been shown to have a variable positive yield of between $2 \%$ and $22 \%,^{5-7}$ Some have even advocated a third angiogram if the first two are negative. Even with a negative initial angiogram, some neurosurgeons would still be prepared to perform an exploratory craniotomy looking for an aneurysm based solely
Table II. Non-aneurysmal causes of subarachnoid haemorrhage

1. Non-aneurysmal perimesencephalic subarachnoid haemorrhage (NPSAH)

2. Pial vascular malformation

3. Dural vascular malformation

4. Occult vascular malformation (cavernoma, capillary telangiectasia)

5. Vasculitis

6. Coagulopathy

7. Intracranial arterial dissection

8. Trauma

9. Intracranial neoplasia

10. Central venous or dural sinus thrombosis

11. Cerebral infarction

12. Pituitary apoplexy

13. Cocaine abuse

14. Spinal tumours and vascular malformations

15. Sickle cell disease

16. Primary infundibular rupture on the pattern of localisation of blood within the subarachnoid space on sectional imaging (CT or MRI) ${ }^{8,9}$ An interesting subgroup of cases with angiographically negative $\mathrm{SAH}$ are those with the subarachnoid blood localised within the perimesencephalic and prepontine and ambient cisterns and repeat negative angiograms. These patients rarely develop vasospasm and universally have a benign clinical course and overall excellent outcome. This subset of cases are deemed to have 'perimesencephalic non-aneurysmal subarachnoid haemorrhage' (PNSAH), first described by Van Gijn and co-workers in $1985 .{ }^{10}$ The cause for these particular haemorrhages is unknown. Speculated mechanisms include vertebrobasilar dissection, rupture of a traversing mesencephalic or pontine perforating artery, rupture of a prepontine vein or bleeding from a small subpial vascular malformation.

Up to $30 \%$ of patients with aneurysmal $\mathrm{SAH}$ have multiple intracranial aneurysms seen at angiography. ${ }^{11}$ Identification of the aneurysm with the highest probability of rupture can be assessed from the pattern of localisation of blood in the subarachnoid space on CT or MR..$^{12-14}$ Angiographic features that allow prediction of the ruptured aneurysm are listed in Table III..$^{15-17}$

In our case the position of the two aneurysms identified on the initial angiogram did not correlate with the localised haemorrhage seen on the CT study. Although asked to embolise the basilar aneurysm, we first performed a full follow-up 4-vessel study during which we identified the recannalised right-sided Al aneurysm. Had this recannalisation not occurred by the time of the second angiogram, an 
Table III. Angiographic features suggesting the ruptured aneurysm in the setting of acute SAH with multiple aneurysms

1. Size: The largest is usually the one that has bled.

2. Contour: The greater the irregularity of the wall of the aneurysm, including 'nipples' or 'daughter domes'.

3. Location: Aneurysms of the anterior communicating artery, basilar tip and posterior inferior cerebellar artery are more likely to bleed.

4. Contrast extravasation: An extremely rare finding, indicates active bleeding during arteriography.

5. Adjacent arterial spasm: Worse around the aneurysm that bled.

6. Reappearance of a previously occult aneurysm.

unnecessary invasive procedure may have been performed on the wrong aneurysm, although as it transpired the basilar aneurysm was in any event unsuitable for endovascular treatment (Fig. 2e). The lesson learnt from this case is that in the event of lack of correlation between the location of a subarachnoid imaging and the location of the responsible aneurysm in the setting of multiple aneurysms, a more intensive or prolonged search for the guilty aneurysm should be performed, possibly even entailing exploratory surgery if repeated cerebral angiograms prove negative.

\section{References}

1. Schwartz TH, Salomon RA. Perimesencephalic non-aneurysmal subarachnoid haemorrhage: review of the literature. Neurosurgery 1996; 39: 433-440.

2. Rinkel GJE, Van Gijn J, Wijdicks EFM. Subarachnoid haemorrhage without detectable aneurysm - a review of the causes. Stroke 1993; 24: 1403-1409.

3. Hamilton MG, Dold ON. Spontaneous disappearance of an intracranial aneurysm after subarachnoid haemorrhage. Can J Neurol Sci 1992; 19:389-391.

4. Spetzler RF, Winestock D, Newton HT, Boldrey EB. Disappearance and reappearance of cerebral aneurysm in serial arteriograms. Case Report. J Neurosurg 1974; 41: 508-510.

5. Duong H, Melancon D, Tampieri D, Ethier R. The negative angiogram in subarachnoid haemorrhage. Neuroradiology 1996; 38: 15-19.

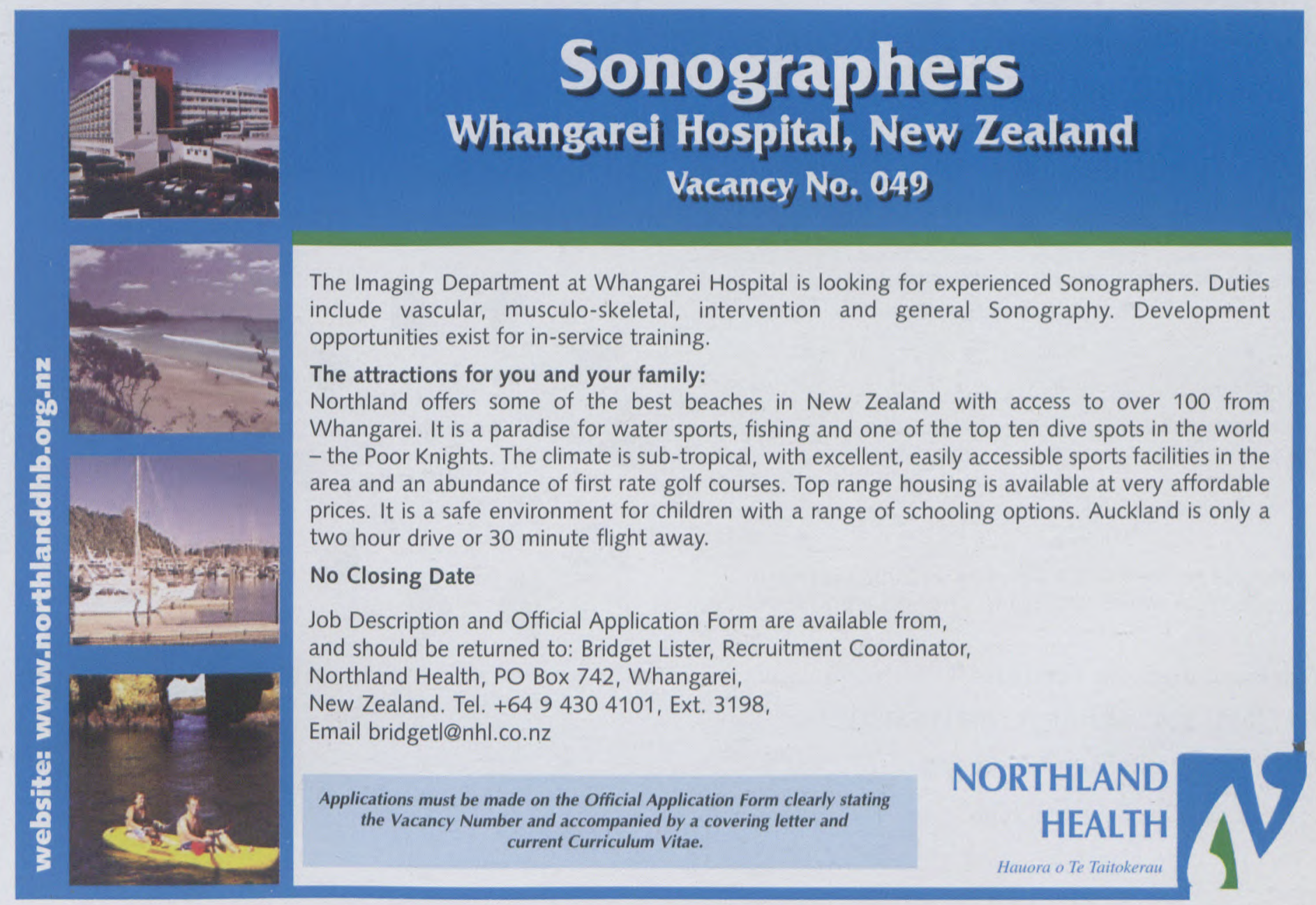


6. de Mesnil de Rochemont R, Heindel W, Wesselmann C, et al. Nontraumatic subarachnoid haemorrhage. Value of repeat angiography. Radiology 1997; 202: 798-800.

7. Urbach H, Zentnet J, Solymosi L. The need for repeat angiography in subarachnoid haemorrhage. Neuroradiology 1998; 40: 6-10.

8. Tatter SB, Cromwell RM, Ogilvy CS. Aneurysmal and microaneurysmal 'angiogram-negative' subarachnoid haemorrhage. Neurosurgery 1995; 37: 48-55.

9. Schievink WI, Wijdicks EF, Piepgras DG, Nichols DA, Ebersold MJ. Perimesencephalic subarachnoid haemorrhage: Additional perspectives from four cases. Stroke 1994; 25: 1507 1511.

10. Van Gijn J, Von Dongen KJ, Vermeulen M, Hijdra A. Perimesencephalic haemorrhage: A non aneurysmal and benign form of subarachnoid haemorrhage. Neurology 1985; 35: 493497.

11. Rinne J, Hernesniemi J, Puranen M, Saari T. Multiple intracranial aneurysms in a defined population: Prospective angiographic and clinical study. Neurosurgery 1994; 35: 803-808.

12. Smaltino F, Bernini FP, D'Auria T. Computed tomography in subarachnoid haemorrhage provoked by multiple aneurysms. Radiol Med $1979 ; 65: 515-519$

13. Van Gijn J, Van Dongen KJ. Computed tomography in the diagnosis of subarachnoid haemorrhage and ruptured aneurysm. Clin Neurol Neurosurg 1980; 82: 11-24.

14. Hackney DB, Lesnick JE, Zimmerman RA, Grossman RI, Goldberg HI, Bilaniuk LT. MR identification of bleeding site in subarachnoid haemorrhage with multiple intracranial aneurysms. J Comput Assist Tomogr 1980; 10: 878-880.

15. Nehls DG, Flom RA, Carter LP, Spetzler RF Multiple intracranial aneurysms: determining the site of rupture. J Neurosurg 1985; 63: 342348 .

16. Wood EH. Angiographic identification of the ruptured lesion in patients with multiple intracranial aneurysms. J Neurosurg 1964; 21: 182-198.

17. Marttilla I, Heiskanen O. Value of neurological and angiographic signs as indicators of the ruptured aneurysm in patients with multiple intracranial aneurysms. Acta Neurochir 1970; 23: $95-102$.

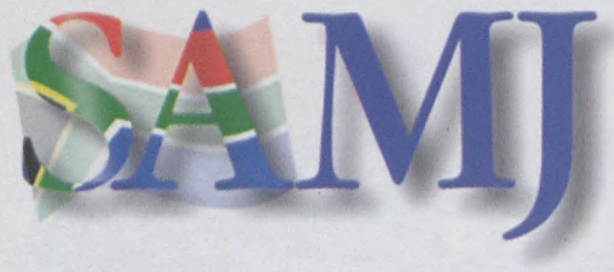

\section{Professional Advertising Rates 2004}

Professional Advertising costs are net and do not include agency commission. Where applicable agencies should add the commission fees to the rates below.

\begin{tabular}{|c|c|c|c|}
\hline Size & $B \& W$ & Spot Colour & Full Colour \\
\hline $30 \times 84 \mathrm{~mm}$ & R 455 & R $\quad 667$ & R 1080 \\
\hline $60 \times 84 \mathrm{~mm}$ & R 1172 & R 1594 & R 2478 \\
\hline $90 \times 84 \mathrm{~mm}$ & R 2514 & R 3526 & $R 5556$ \\
\hline$Q / P$ & R 3326 & R 5784 & R 7046 \\
\hline $\mathrm{H} / \mathrm{P}$ & R 4801 & R 7255 & $R 9671$ \\
\hline$F / P$ & R 8226 & $R 10673$ & R 12828 \\
\hline
\end{tabular}

Rates effective from 1 December 2003. Rates and publication dates subject to change without notice.

\section{Contact : Vanessa Sampson}

Tel : (021) $5306549 \cdot$ Fax : (021) 5314126

\section{Email : vanessas@samedical.org}

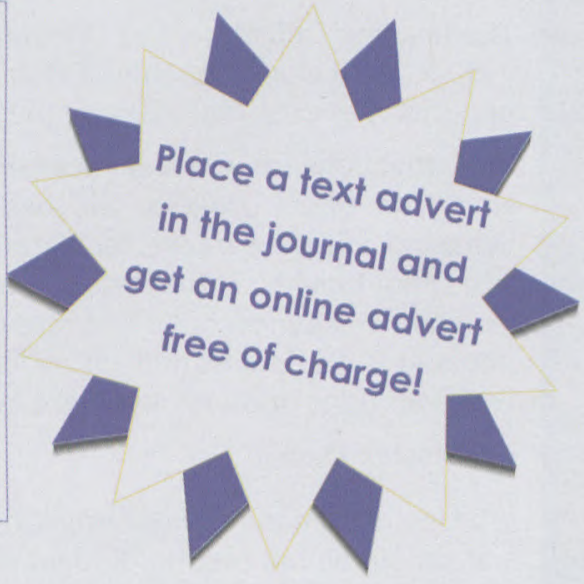

South African Medical Association, Health and Medical Publishing Group, Lonsdale Building, Gardener Way, Pinelands, 7405. 